\title{
Ground-Penetrating Radar Antenna Design for Homogeneous and Low-Loss Dielectric Multilayer Media
}

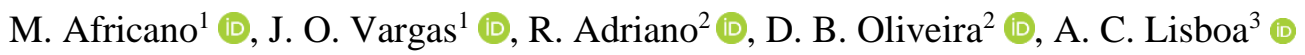 \\ ${ }^{I}$ Graduate Program in Electrical Engineering - Universidade Federal de Minas Gerais - Av. \\ Antônio Carlos 6627, Belo Horizonte, MG, Brazil, mvafricano@ufmg.br, jolgervg@ufmg.br \\ ${ }^{2}$ Department of Electrical Engineering - Universidade Federal de Minas Gerais, rluiz@cpdee.ufmg.br, \\ diogo@cpdee.ufmg.br \\ ${ }^{3}$ GAIA Solutions on Demand - Rua Prof. José Vieira de Mendonça 770, 31310-260, Belo Horizonte, MG, \\ Brazil,adriano.c.lisboa@gmail.com
}

\begin{abstract}
Non-destructive evaluation of multilayer media represents an electromagnetic inverse problem, usually solved with analysis techniques in the time and frequency domains. The timedomain analysis technique depends directly on the accurate detection of reflected peaks measured by ground-penetrating radar (GPR), which limits this technique when the reflected pulses overlap with each other or the transmitted signal. Therefore, this paper presents a new practical methodology to obtain the antenna design requirements (operating frequency and temporal response) that avoid overlapping reflected pulses. The major finding is that the proposed methodology enables the production of specific antennas for the analyzed homogeneous and low-loss dielectric multilayer problem, serving as a practical guide for GPR antenna design. A typical pavement example is analyzed to apply the methodology, and, as a result, an ultra-wideband (UWB) directive antenna with tolerable dispersion is obtained according to the design specifications. Finally, the antenna simulations were compared and validated with measurements.
\end{abstract}

Index Terms - Ground-penetrating radar, Multilayer problem, Pavement, Ricker wavelet, UWB antenna.

\section{INTRODUCTION}

Nondestructive testing (NDT) techniques have emerged with the intention of examining any object, material, or system without affecting its future utility. A well-accepted NDT technique is ground penetrating radar (GPR), which has been widely explored and applied in many areas, such as archaeology, geophysics, and engineering [1]-[3]. The GPR technique represents a faster alternative to assess the interior of different structures and also contributes to their preservation.

Several structures, such as railways [4], pavements [5], bedrock and retaining walls [1] can be modeled as multilayer media and analyzed using GPR. In broad terms, GPR detects the reflections originating from electromagnetic discontinuities on the structure. In this way, the information obtained by GPR can be used to determine the characteristics of each layer, e.g. thickness and relative electric permittivity. This process represents an inverse electromagnetic problem that can be solved using two broad approaches: analysis techniques in the frequency and time domains [6]. Nevertheless, 
choosing a solution technique for the inverse problem has a direct impact on the GPR operating frequency, and, consequently, the antenna to be used.

Frequency-domain analysis techniques usually utilize optimization tools to solve the electromagnetic inverse problem [7], [8]. However, defining the optimization problem based on the reflected electric-field phasor is a challenge, because the uniqueness of the solution must be guaranteed. This means that the objective function must be ensured to have only a unique minimum or maximum point. In a comprehensive study of this problem, Oliveira et al. [8] presented a methodology that ensures smoothness in the error function of the inverse problem to facilitate its solution. In contrast, the proposed methodology imposes restrictions on the GPR operating frequency, which will limit the minimum size of the antenna. Furthermore, the findings in [8] suggest working with narrow-band antennas, which have an analytic closed-form that facilitates the design.

In contrast, time-domain analysis techniques generally use the travel time of the electromagnetic wave between the layers and the reflected peak amplitude to define the layers' dielectric properties and thicknesses [9], [10]. This analysis technique uses GPR measurements, easy calculations and two assumptions to solve the inverse problem: propagation in (a) low-loss dielectric, i.e., conductivity $\sigma \ll$ $\omega \varepsilon$, where $\omega$ is the angular frequency and $\varepsilon$ is the permittivity; and (b) homogeneous layers, which means that the dielectric constant is assumed to be equal throughout the layer. This time- domain analysis approach, based on travel time through the layers and presented in detail in [9], will be considered for this study. Nevertheless, this method may become inaccurate if inadequate reflection detections (due to overlapping) are performed. To avoid this drawback, a good vertical resolution is necessary. Consequently, there is a need to design antennas with the ability to transmit and receive short pulses with low distortion.

It is believed that the most significant advances in a GPR system can be achieved with the antenna design (transmitter-receiver). In this regard, a complete review of GPR antenna characteristics is presented in [11]. Several studies with specific applications for GPR have been developed to seek antenna systems with optimal designs and low cost [12], [13]. On this matter, patch antennas have reached increasing prominence by achieving versatile solutions in multiple applications where a radiant element is required [14]-[16].

For instance, authors in [17] proposed using multi-objective optimization to design 3 antennas with applications in 3 different areas, including GPR. In that research, the solution of contradictory objectives related to directivity, impedance matching, cross polarization, and frequency range using stochastic and deterministic algorithms in the solution was carried out. To find improvements over traditional approaches that only use spectral analysis to optimize antennas, a multi-criteria optimization approach was proposed directly in the time domain [18] for a bow-tie antenna. The results demonstrated a good balance between radiation and admittance matching in the analyzed frequency range. In addition, another interesting analysis was suggested in [19] to design an optimized antenna that adapts to specific input signals. They ensured a high correlation between the Brazilian Microwave and Optoelectronics Society-SBMO received 14 June 2019; for review 19 June 2019; accepted 18 Feb 2020 Brazilian Society of Electromagnetism-SBMag 
input signal in the time domain and the output signal in the receiving antenna. Further, the authors in [19] achieved not only a minimum voltage standing wave ratio (VSWR), but also low temporal dispersion.

Although previous studies have proposed antennas for general GPR applications, none of them are based on the specific problem to establish the antenna-design characteristics, such as the central frequency, bandwidth, and temporal response. Therefore, we propose a practical methodology to obtain the specific antenna-design requirements that allow problems modeled as multilayer media to be solved with time-domain solution techniques while avoiding pulse overlapping. In other words, we suggest correlating, in an unequivocal (analytic-direct) way, some a-priori physical knowledge of the multilayer problem with the antenna characteristics to be designed. This a-priori knowledge means that some typical estimates are known for the relative electric permittivity and thickness of layers, which are generally obtained through other techniques. In this sense, the methodology presented in this paper represents a practical guide for GPR antenna design, enabling the production of a specific antenna for any analyzed multilayer problem. Consequently, this guide can contribute to obtaining dedicated GPR equipment.

The paper is organized as follows: Section II presents the step by step formulation to obtain the spectral and temporal antenna design requirements. Then, Section III contains a description of a simple multilayer medium used to test the methodology proposed in Section II. Later, Section IV explains the ultra-wideband (UWB) antenna selection and parametric analysis to fulfill the requirements obtained for the test problem in Section III. Finally, the results are presented and discussed in Section V, and Section VI lists the conclusions of the work

\section{Proposed Methodology}

As was pointed out in the introduction, the antenna design is dependent on the GPR operating frequency, which in turn depends on the type of analyzed multilayer structure and the inquiring signal. From this correlation, the following methodology was developed. The methodology starts with the analysis of the inquiring GPR signal. Then, some information from the time-domain inverse problem solution is extracted, and finally, a signal-overlap criterion is established according to [20]. Following these methodology steps, the requirements for antenna design can be obtained for a specific problem. Thus, if an antenna meets those requirements, the overlapping reflected pulses would be avoided. In this way, the proposed methodology serves as a practical guide in the design of GPR antennas.

\section{A. Ricker wavelet}

The Ricker wavelet is the pulse selected for use in the proposed methodology. It is frequently used in GPR applications [21], [22] because it solves the problem of low-frequency components (spectrum near DC) presented in the Gaussian pulse.

The equation that describes Ricker wavelet is as follows: 


$$
x_{r}(t)=A\left[1-\frac{2}{\tau^{2}}\left(t-t_{0}\right)^{2}\right] \exp \left\{\frac{-\left(t-t_{0}\right)^{2}}{\tau^{2}}\right\}
$$

where $A$ is the amplitude, $t_{0}$ is the time shift, and $\tau$ is the time constant.

The Fourier transform amplitude $\left|X_{r}(f)\right|$ of the Ricker wavelet is given in Eq. (2).

$$
\left|X_{r}(f)\right|=A(2 \pi f)^{2} \exp \left\{-\frac{1}{4}(2 \pi f \tau)^{2}\right\}
$$

The peak emission frequency $f_{p}$ can be found by deriving Eq. (2) and equating it to zero. As a consequence, the peak emission frequency should satisfy $\left(2 \pi f_{p}\right)^{2}=4$. By isolating $f_{p}$ from this term and assuming that the pulse width is $t_{p} \approx 6 \tau$, Eq. (3) is obtained.

$$
f_{p}=\frac{6}{\pi t_{p}}
$$

As shown, it is possible to define, through Ricker wavelet analysis, the peak emission frequency $f_{p}$. This frequency represents the antenna's central frequency, which is an important parameter in the antenna design. As can be seen in Eq. (3), the pulse width $t_{p}$ is still an unknown variable which will be obtained in the next methodology step.

\section{B. Impacts of selecting the inverse problem solution approach}

Selecting an approach (either in the time or frequency domain) to solve the inverse problem imposes a set of restrictions leading to different GPR operating frequencies. The time-domain analysis approach uses a short pulse to feed the GPR antenna, which results in a spectrum rich in frequency components. The pulse width in this technique is determined by the analyzed multilayer structure.

In the time-domain analysis approach, an adequate identification of each reflected pulse is necessary because the layers' characteristics, such as thickness and relative electric permittivity, are obtained from the time between multiple pulse reflections and their amplitudes [9]. In this way, the total time of the incident pulse for each interface should be less than the time for the transmitted wave portion to travel through the next layer, reflect on the next interface, and return.

The intersection of subsequent pulses will be null if

$$
t_{p} \leqslant 2 \Delta t_{d_{i}}, \forall_{i}=0,1, \ldots, n,
$$

where $t_{p}$ is the Ricker wavelet width, $\Delta t_{d_{i}}$ represents the required time for the wave to travel through layer $d_{i}$, and $n$ is the number of layers in the problem. The time that the pulse takes to travel through each layer $\left(\Delta t_{d_{i}}\right)$ is obtained by

$$
\Delta t_{d_{i}}=\frac{d_{i} \sqrt{\varepsilon_{i}}}{c}, \forall_{i}=0,1, \ldots, n,
$$

where $\varepsilon_{i}$ and $d_{i}$ are the relative electric permittivity and thickness of layer $i$, respectively, and $c$ is the speed of light. Thus, by substituting Eq. (5) in Eq. (4), the general equation for any multilayer problem is obtained

$$
t_{p} \leqslant 2\left(\frac{d_{i} \sqrt{\varepsilon_{i}}}{c}\right), \forall_{i}=0,1, \ldots, n .
$$

It is evident from (6) that both the thinnest layer thickness and the lowest permittivity determine the selection of $t_{p}$. In this way, the pulse is defined in terms of the relative electric permittivity and Brazilian Microwave and Optoelectronics Society-SBMO received 14 June 2019; for review 19 June 2019; accepted 18 Feb 2020 
thickness of each pavement layer using some a-priori knowledge about the analyzed structure, as will be explained in the next section.

Now, the appropriate requirements for designing the antenna from the analyzed multilayer structure can be completely defined. First, the peak emission frequency $f_{p}$ can be calculated using Eq. (3) where $t_{p}$ is the pulse width satisfying Eq. (6). Then, the cut-off frequencies and bandwidth can be defined for $f_{p}$. These frequencies are commonly selected depending on the application. For instance, ANSI STD C63.10-2013 [23] and IEEE STD 686-2017 [24] define for UWB and radar devices respectively that the cut-off frequencies $f_{L}$ and $f_{H}$ are the lowest and highest frequencies where the signal is at least $10 \mathrm{~dB}$ below the peak power level. On the other hand, circuit theory commonly defines these frequencies when the peak power falls at half $(-3 \mathrm{~dB})$ [25]. For this work, the half-width cut-off frequencies at $-6 \mathrm{~dB}$ were used because it allows a pulse reconstruction closer to the Ricker wavelet. Thus, the approximations for the frequency-domain parameters of the Ricker wavelet as a function of $f_{p}$, as determined by Wang in [26], are as follows:

$$
\begin{aligned}
f_{C} & \approx 1.059095 f_{p}, \\
f_{L} & \approx 0.481623 f_{p} \\
f_{H} & \approx 1.636567 f_{p}, \\
B W & \approx 1.154944 f_{p},
\end{aligned}
$$

where $f_{c}$ is the central frequency, $f_{L}$ and $f_{H}$ are the lower and upper cut-off frequencies, respectively and $B W$ is the bandwidth. These specifications guarantee that there will not be any pulse overlap which contributes to the accuracy of the solution technique of a GPR inverse multilayer problem. In summary, the antenna to be used in GPR equipment must be capable of transmitting a Ricker wavelet respecting the characteristics described by Eqs. (3) and (7)-(10).

\section{Pulse overlap criterion}

The spectral and temporal antenna characteristics were already obtained in Subsections II.A and II.B. Nevertheless, antennas are inherently dispersive elements and, therefore, can cause undesired reflections. It is important to know which is the antenna's permissible dispersion $\left(t_{p_{\max }}\right)$ that still allows separation of the reflections originating from pavement discontinuities. For this, the criterion proposed by Jol [20] for a Gaussian pulse was used and adapted for Ricker wavelet.

Fig. 1 shows the extremes for two Ricker wavelets, in which the pulse is characterized by the width at half energy $W$ defined by the time difference between the extremes of the pulse when they achieve half of the maximum value of energy. Fig. 1(a) depicts pulses that are clearly separable without any overlap. In turn, Fig. 1(b) presents pulses that overlap but are still considered distinguishable (separable). According to [20], two Gaussian pulses are distinguishable if they are separated by at least the width at half amplitude. However, for the Ricker wavelet, the width at half energy is used instead of that at half amplitude, as shown in Fig. 1.

Therefore, if certain pulses are allowed to overlap by the width at half energy $T \approx W$, the restriction of the pulse can now be given by

Brazilian Microwave and Optoelectronics Society-SBMO received 14 June 2019; for review 19 June 2019; accepted 18 Feb 2020 Brazilian Society of Electromagnetism-SBMag 
where

$$
W \leqslant 2 \Delta t d_{i}, \forall_{i}=0,1, \ldots, n
$$

$$
W \approx \frac{t_{p_{\max }}}{5}
$$

By substituting Eq. (12) into (11) and using (5), the allowed maximum pulse width is given by

$$
t_{p_{\text {max }}}=10 \frac{d_{i} \sqrt{\varepsilon_{i}}}{c}, \forall_{i}=0,1, \ldots, n .
$$

As a result, the dispersive characteristic of the antenna is defined by the separation threshold between two pulses.

Thus, given an estimate of the multilayer problem to be assessed, the design antenna requirements can be defined using equations (7)-(10) and (13), as presented in Section III.

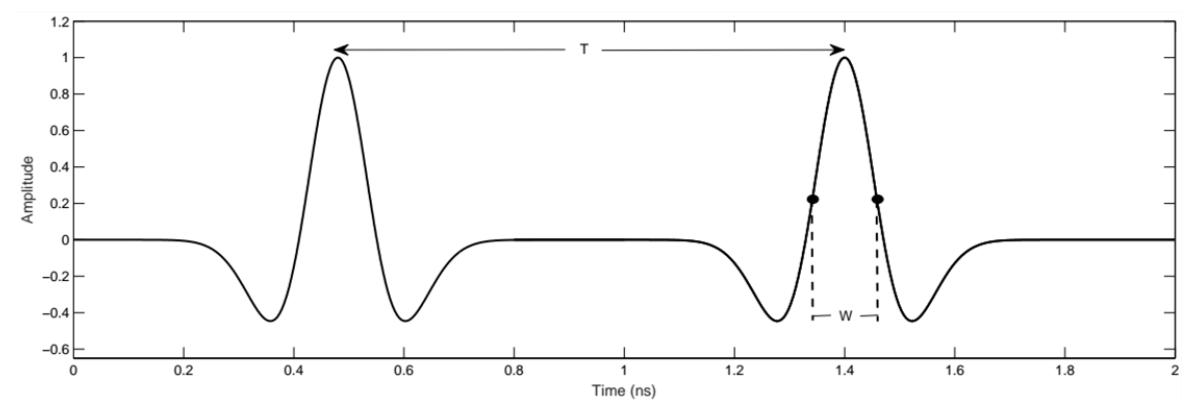

(a) Clearly separated $(\mathrm{T}>\mathrm{W})$

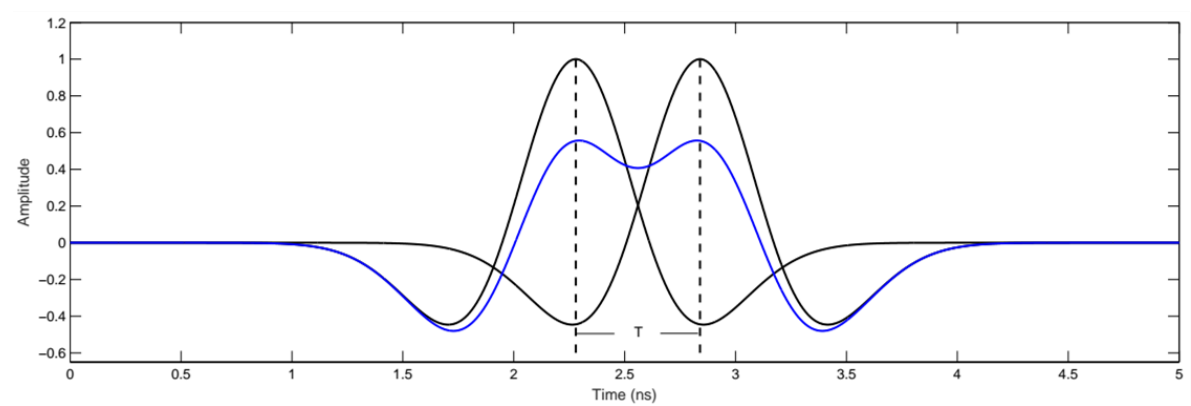

(b) Still distinguishable $(\mathrm{T} \approx \mathrm{W})$

Fig. 1. Overlap criterion of Ricker wavelets in the time domain.

\section{Test Problem}

A multilayer structure was selected in this work to show how the methodology proposed in Section II can be used. GPR has been used commonly as an indirect measurement for construction and maintenance of highways [27], where pavement can be modeled by multiple overlapping layers. This topic has been widely explored and much research has been carried out, such as [28], [29].

The three-homogeneous-layer model presented in Fig. 2 was used as a test pavement problem. In Fig. 2, Tx and $\mathrm{Rx}$ represent the transmitter and receiver antennas, respectively, $d_{0}$ is the distance between the pavement surface and the antennas. $d_{1}$ and $d_{2}$ are the unknown layer thicknesses; $\varepsilon_{1}, \varepsilon_{2}$ and $\varepsilon_{3}$ are the unknown relative electric permittivities related to each layer ( 1 for asphalt, 2 for the base and 3 for subgrade layer). 


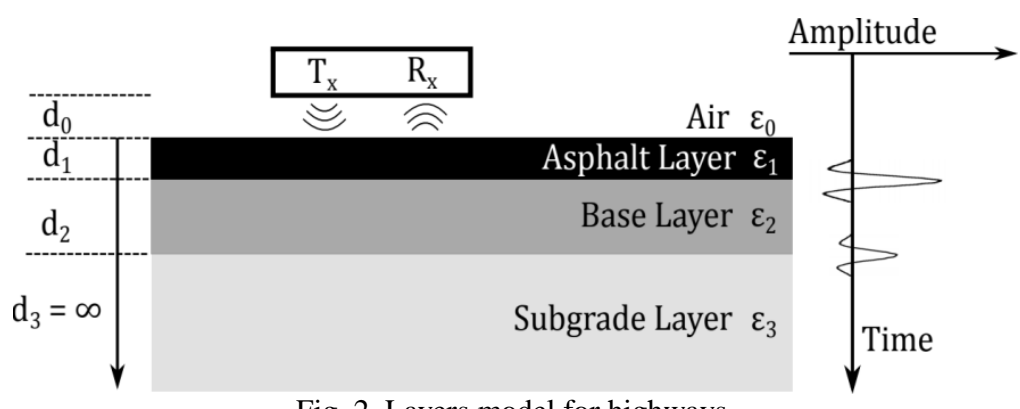

Fig. 2. Layers model for highways.

To apply the proposed methodology described in the previous section, the first step is to calculate the Ricker wavelet width by using Eq. (6). This equation is dependent on $\varepsilon_{r_{i}}$ and $d_{i}$, which are unknown parameters (they are predicted with GPR testing). Then, some a-priori knowledge of the range of possible values of the electromagnetic properties and thicknesses of the pavement layers needs to be considered here. This interval can be defined using some direct measurements in the tested multilayer media using destructive techniques or it can also be defined using the results of typical pavement values of permittivities and thicknesses, which were measured or estimated in previous works [27], [30], [31]. To the test problem proposed in this paper, the range of the permittivity and thickness are shown in Table I [27], [30], [31].

The most restrictive case was selected from Table I to generate a worst-case analysis. This means that the smallest values of both permittivity and thickness of each layer in Table I were used to calculate $t_{p}$, and, from these $t_{p}$ values, the shortest travel time was selected. In this way, the nonoverlap in the GPR reflected pulses will be ensured, regardless of the real value of the pavement parameters. Thereby, the pulse width of Ricker wavelet was calculated for each layer using Eq. (6), and the obtained values are listed in Table I.

TABLE I. ESTIMATED VALUES OF TYPICAL LAYERS’ PARAMETERS

\begin{tabular}{lccc}
\hline Layer & $\varepsilon_{r_{i}}$ & $d_{i}(\mathbf{c m})$ & $t_{p}(\mathbf{n s})$ \\
\hline 1. Asphalt & $3-12$ & $6-10$ & 0.69 \\
2. Base & $5-10$ & $20-30$ & 2.98 \\
3. Sub-grade & $10-25$ & -- & -- \\
\hline
\end{tabular}

It can be defined from the data in Table I that if the pulse width is at most $0.69 \mathrm{~ns}$, the overlap between layers reflections for the pavement problem would be avoided. Thus, it is demonstrated that the pulse was actually defined based on a-priori pavement physical knowledge assuming a worst-case scenario.

The next step is to calculate the peak emission frequency $\left(f_{p}\right)$ using Eq. (3) where $t_{p}$ is the one obtained for the thinnest layer in Table I $\left(t_{p}=0.69 \mathrm{~ns}\right)$. Then, the antenna design values were calculated using Eqs. (7)-(10). Thus, all the antenna requirements were estimated; they are presented in Table II.

Finally, the maximum antenna permissible dispersion $\left(t_{p_{\max }}=3.46 n s\right)$ was also calculated for the thinnest layer (asphalt) using Eq. (13). 
TABLE II. ANTENNA FREQUENCY DESIGN REQUIREMENTS FOR THE THREE LAYER MODEL.

\begin{tabular}{cc}
\hline Parameter & Value $(\mathbf{G H z})$ \\
\hline$f_{p}$ & 2.77 \\
$f_{c}$ & 2.93 \\
$f_{L}$ & 1.33 \\
$f_{H}$ & 4.53 \\
$B W$ & 3.2 \\
\hline
\end{tabular}

\section{UWB ANTENNA}

Once the spectral and temporal requirements are obtained for the test problem using the proposed methodology in Section III, an antenna topology should be selected. In general, an antenna for GPR applications needs to consider the following characteristics: wide frequency bandwidth to obtain high resolution, good gain, radiation efficiency, good impedance matching, low dispersion, small size and low weight [11]. Many GPR-UWB antennas have been studied in the literature that could meet the obtained requirements: bowtie antennas [32], [33], spiral slot antennas [34], circular antennas [35], Vivaldi antennas [15], resistive linear antennas [36], among others [11].

For this study, the selected microstrip patch antenna geometry was inspired from [37], as it presents a simple geometry for construction, ease of parametrization, and low implementation cost. Therefore, it represented a good starting point for a parametric study. The antenna in [37] has a gain that varies from 3 to $6 \mathrm{~dB}$ and the bandwidth from 3.6 to $12 \mathrm{GHz}$, which means that it is not in the desired frequency band. Besides, it has an omnidirectional radiation pattern. To solve this, a reflector plane was added behind the antenna and a parametric study was carried out, as shown in Fig. 3.

Fifteen parameters were used to describe the antenna geometry: the width $w_{d}$ and length $L_{d}$ of the dielectric substrate, the width $W_{f}$ and length $L_{f}$ of the feed line, the width $W_{p}$ and length $L_{p}$ of the patch, the width $W_{g}$ and length $L_{g}$ of the ground plane, the position on the $\mathrm{x}$ - and $\mathrm{y}$ - axes of one of the arms $\left(x_{a}, y_{a}\right)$, and the width $w_{x a}$ and length $L_{y a}$ of the arm. To reduce the search space, the dimensions of the other arm were obtained assuming that the antenna is symmetrical. Also, the width $W_{R}$ and length $L_{R}$ of the reflector plane, and the distance $D_{R}$ between the reflector plane and the antenna were considered. The dielectric substrate thickness was defined as a constant $(h=1.6 \mathrm{~mm})$.

\section{RESULTS AND DISCUSSION}

\section{A. Parametric analysis of the antenna}

A parametric analysis of the proposed antenna that aims not to distort the Ricker wavelet was performed. This procedure resulted in the antenna parameters listed in Table III. 


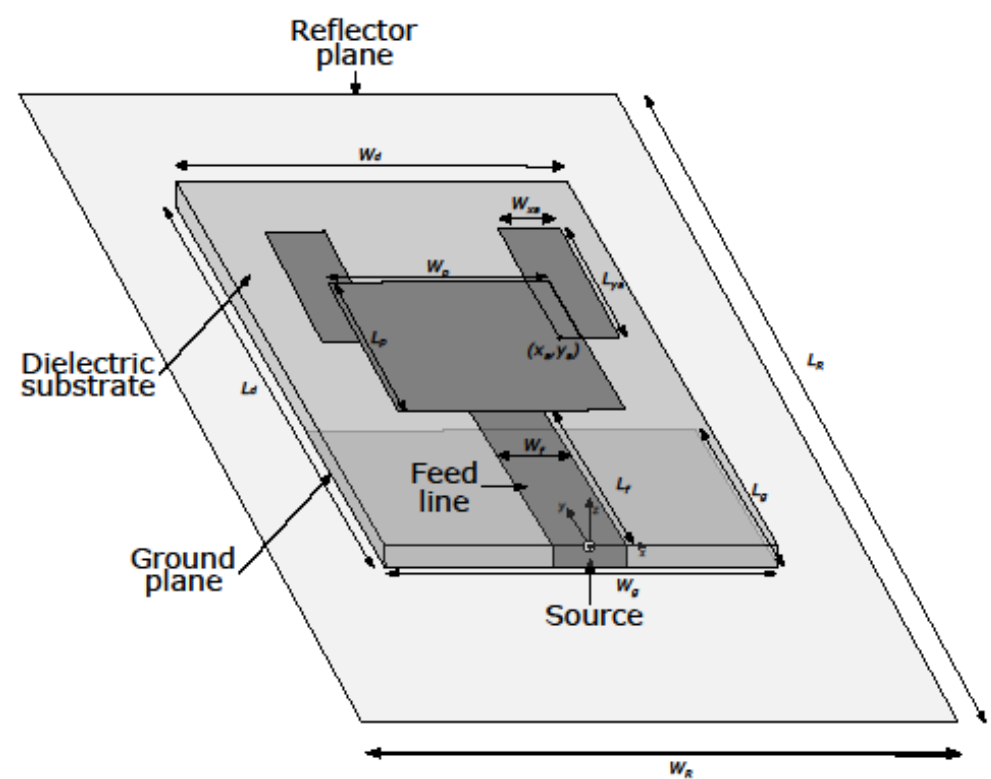

(a) Perspective view

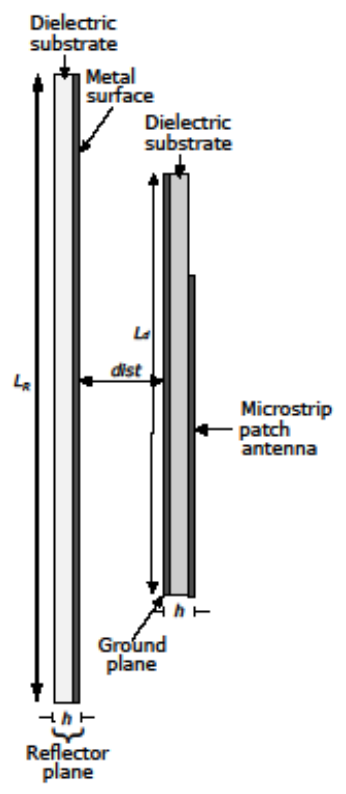

(b) Cross-section view

Fig. 3. U-patch antenna geometry parameterization.

TABLE III. NEW ANTENNA PARAMETERS OBTAINED FROM THE PARAMETRIC ANALYSIS

\begin{tabular}{cc}
\hline Parameter & Value (mm) \\
\hline$W_{d}$ & 47.9 \\
$L_{d}$ & 78.5 \\
$W_{f}$ & 2.54 \\
$L_{f}$ & 28.5 \\
$W_{p}$ & 13.3 \\
$L_{p}$ & 35.3 \\
$W_{g}$ & 35 \\
$L_{g}$ & 25.3 \\
$x_{a}$ & 6.99 \\
$y_{a}$ & 31.2 \\
$W_{x a}$ & 16.9 \\
$L_{x a}$ & 24.4 \\
$W_{R}$ & 90 \\
$L_{R}$ & 120 \\
$D_{R}$ & 22.5 \\
\hline
\end{tabular}

\section{B. Simulation of antenna performance}

The new antenna geometry was simulated in High-Frequency Structure Simulator (HFSS) where the microstrip line, patch, arms, ground, and reflector plane were modeled as perfect electric conductors (PECs), and a port was connected to the microstrip line as the feed. The material selected for the substrate was FR-4 $\left(\tan \delta=0.02, \varepsilon_{r}=4.4\right)$. The designed antenna with the reflector plane was simulated and analyzed (see Fig. 4).

The 3D radiation pattern simulated in the central operating frequency $(3 \mathrm{GHz})$ for the antenna is presented in Fig. 4(a). It can be observed from Fig. 4(a) and Fig. 4(c) that the inclusion of the reflector plane made the radiation pattern directive (as expected) not only at central frequency but in the complete bandwidth. On the other hand, the VSWR presented an increase at low frequencies of the 
analyzed band compared with the original reference (see Fig. 4 (b)). However, this does not represent a drawback, because the most important antenna parameter to be guaranteed in the GPR inverse problem solution technique is the pulse fidelity rather than the power transmission.

\section{Antenna measurements}

To perform the measurements, a Rohde \& Schwarz vector network analyzer (VNA) with coaxial testing cables was used in a non-anechoic environment. The coaxial cables were properly calibrated using the thru-open-short-match (TOSM) calibration and the ZV-Z135 female calibration kit from Rohde and Schwarz. Finally, the antenna was connected to the coaxial cables using a female SMA$50 \Omega$ connector. The antenna was built on a $47.9 \times 78.5 \times 1.6 \mathrm{~mm}^{3}$ FR-4 substrate and it is shown in Fig. 5. The transmitter-receiver link measurements were performed following the methodology presented in [38], placing two equal antennas separated by a distance considered to be far-field ( $5 \lambda$ of the operating central frequency). At the same time, this link was modeled in HFSS for antenna validation, which comprises comparing the measured results of gain, and transmission losses with the simulated ones.

The S-parameters from the antenna measurement were exported and compared with the simulation ones (see Fig. 6). It is observed from Fig. 6 that the results present a good correlation at frequencies higher than $3 \mathrm{GHz}$. It can also be seen that the reflection coefficient $\left(s_{11}\right)$ is better in measurements within the whole bandwidth. This indicates that the reflection losses are negligible and less than $10 \%$ of the voltage energy returns to the source. Differences in the measured and simulated values presented in Fig. 6 may be due to the non-anechoic environment and uncertainties in the FR-4 electromagnetic properties.

Next, the Friis transmission equation was used as shown in Eq. (14) to calculate the antenna realized gain, where $r$ is the distance between two equivalently designed antennas, $\lambda$ is the free-space wavelength, and $s_{21}$ is the transmission coefficient.

$$
G=\frac{4 \pi r}{\lambda}\left|s_{21}\right|
$$

Fig. 7 shows the gains calculated using Eq. (14) from the HFSS simulated link (dashed line) and the measured link (solid line), comparing them with the HFSS gain for a single antenna (dotted line). This comparison was performed as a validation of the Friis approximation, where the distance between the antennas must be far-field. Thereby, Fig. 7 illustrates that the defined Friis approximation using the distance of $50 \mathrm{~cm}$ provides good results comparing the Friis simulated gain (dashed line) and the HFSS antenna gain (dotted line).

In contrast, gain from the measurements (solid line: Friis measured) was satisfactory at frequencies higher than $3 \mathrm{GHz}$. However, the results differ from simulated ones at lower frequencies. This result was expected because the Friis equation (Eq. (14)) depends on the $s_{21}$ parameter, which has a similar behavior between the simulated and measured results (see Fig. 6). 


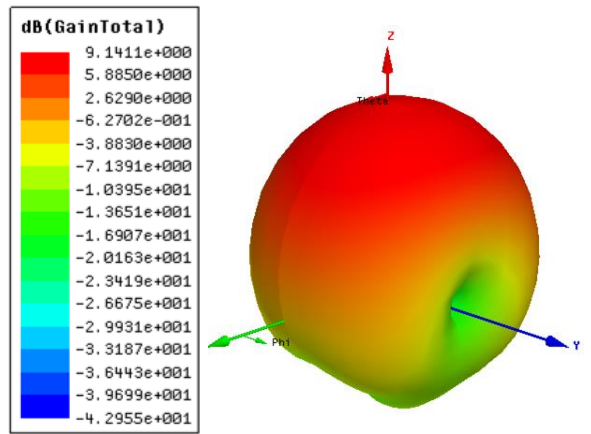

(a) $3 \mathrm{D}$ total gain at $3 \mathrm{GHz}$

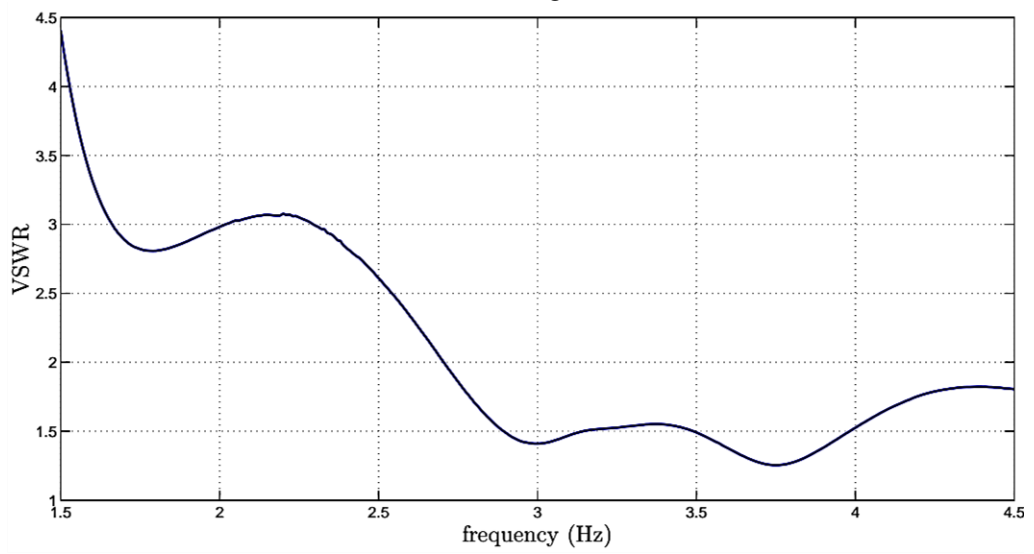

(b) VSWR

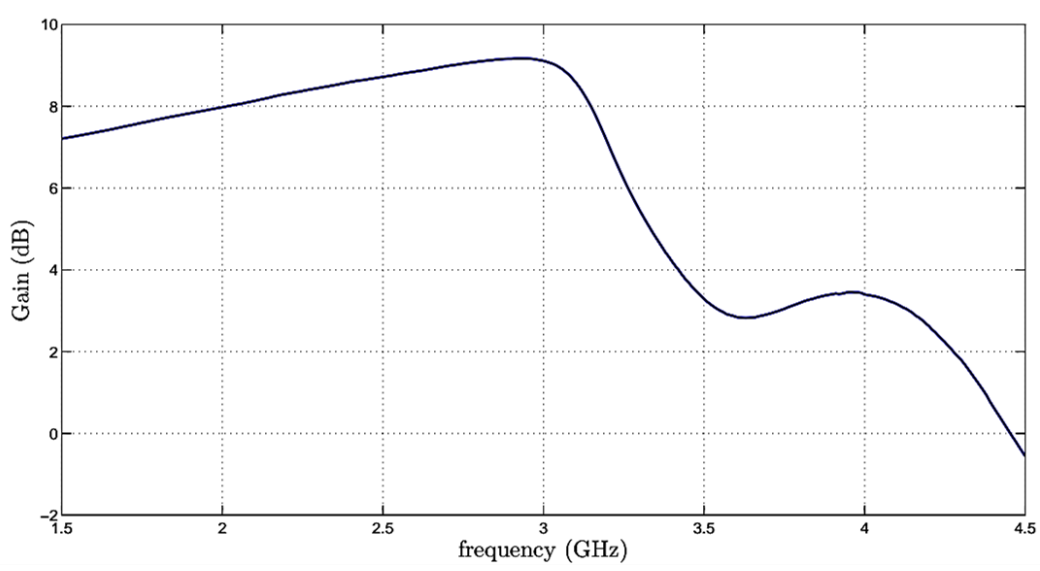

(c) Total gain

Fig. 4. Antenna simulation results.

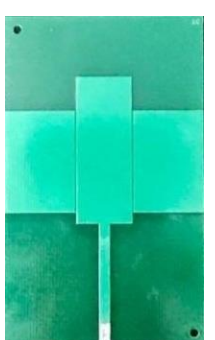

(a) Front view

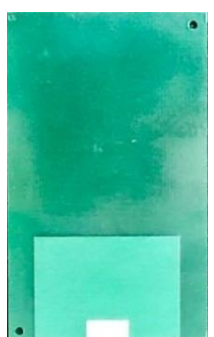

(b) Back view

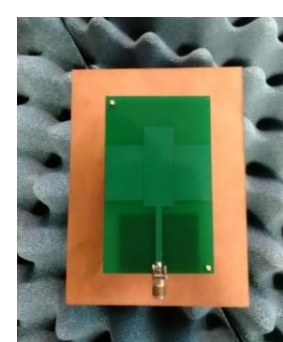

(c) Front view with reflector plane

Fig. 5. Designed antenna. 

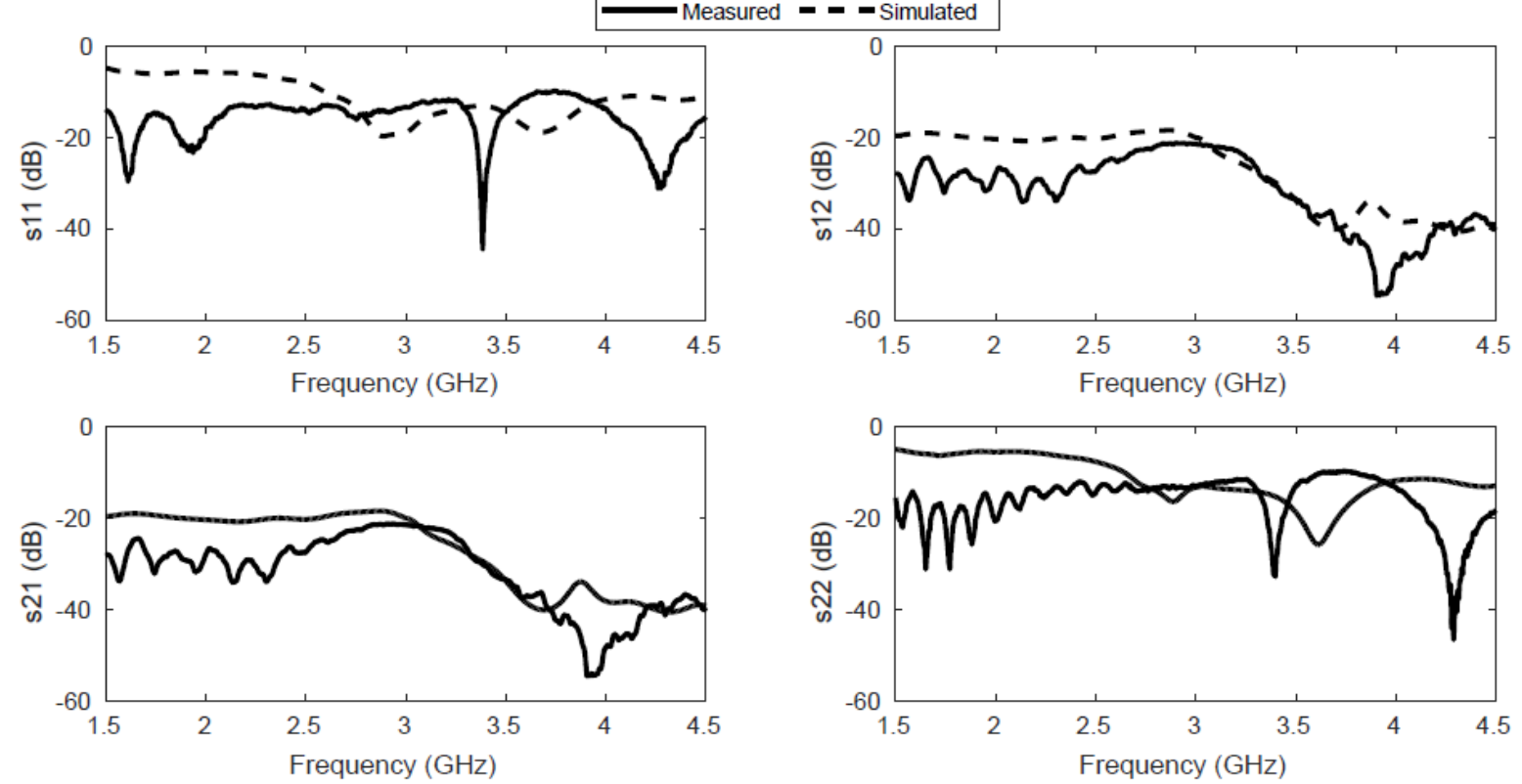

Fig. 6. S-parameters in dB from HFSS simulation and measurements.

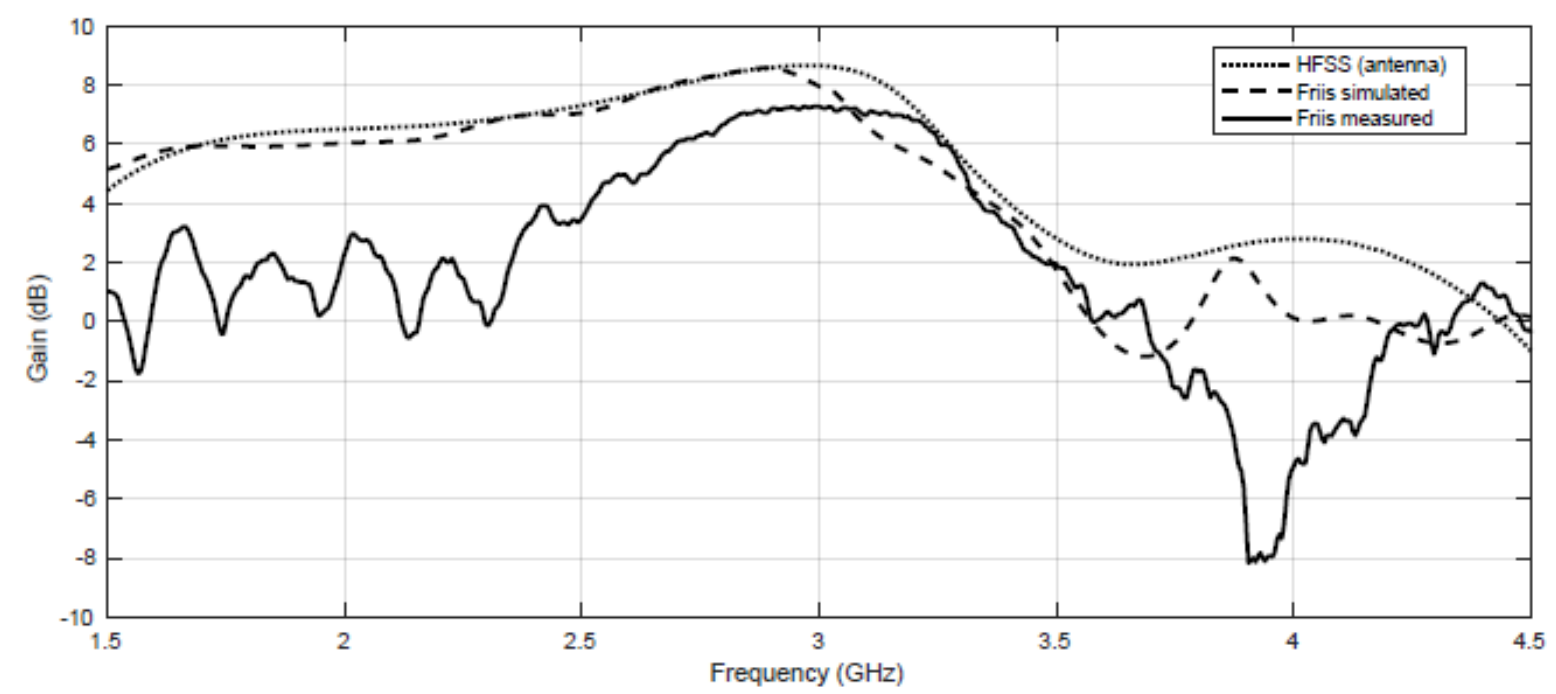

Fig. 7. Realized gain.

\section{Time-domain analysis}

A pulse temporal analysis was performed using the designed antenna to visualize the signal spread.

To achieve this, the S-parameters were exported from HFSS and from measurements to the Advanced Design System (ADS) software, where they are modeled as a two-port black box. Fig. 8 shows the complete simulated circuit of a Ricker wavelet transmission and reception using the designed antenna. The only change between measured and simulated pulses is the black box containing the Sparameters. The Ricker wavelet was imported to ADS from MATLAB with a pulse width $t_{p}=0.69 n s$ and connected to a $50-\Omega$ resistance.

The voltage signal at each node (1-Transmitted, 2-Coupled, 3-Received in Fig. 8) was simulated and the results are presented in Fig. 9. In Fig. 9, small reverberations in the signal coupled to the antennas (dashed line) can be seen. These are due to impedance matching, and therefore, existing reflections are added to the input signal (solid line). The dotted line in Fig. 9 represents the output signal, which was multiplied by a factor (of 50) for visualization purposes.

Brazilian Microwave and Optoelectronics Society-SBMO received 14 June 2019; for review 19 June 2019; accepted 18 Feb 2020 Brazilian Society of Electromagnetism-SBMag 


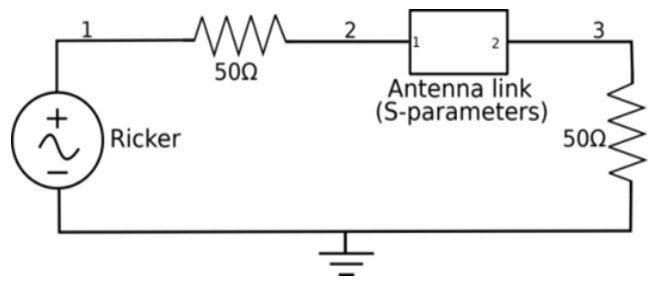

Fig. 8. Implemented circuit in ADS.

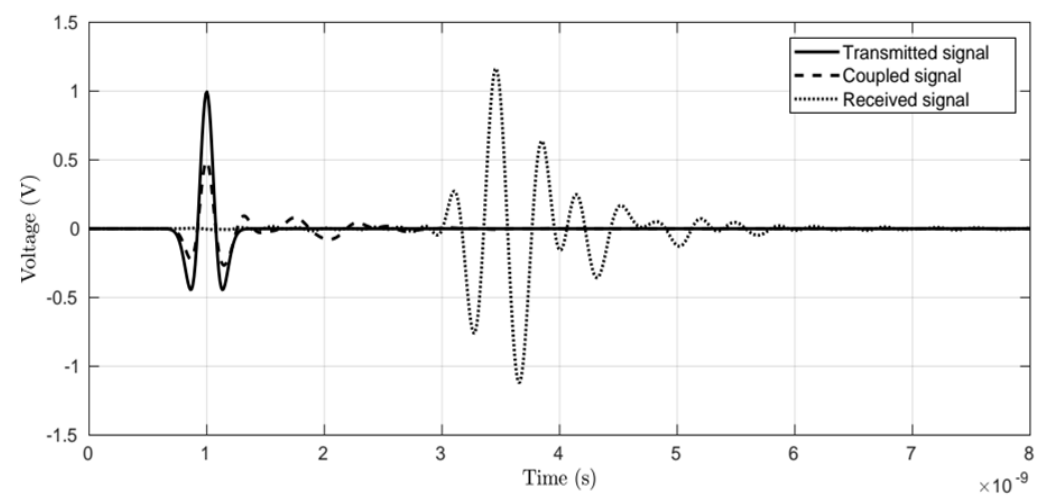

(a) Simulated antenna link

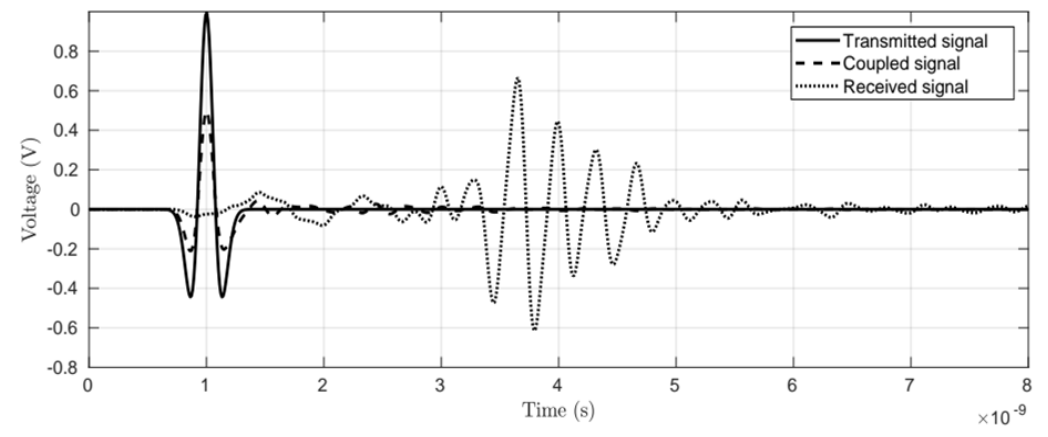

(b) Measured antenna link

Fig. 9. Voltage pulses.

To perform the spread (dispersion) analysis, the power at the system output was analyzed. A shorter spread time indicates that there will be no overlap of the reflected signals. The simulated and measured power graphs from the transmitted and received signals are shown in Fig. 10. Table IV contains the total pulse duration at the output $t_{p_{\text {spread }}}$ when the system was excited with the Ricker wavelet.

As demonstrated in Table IV, the pulse width obtained from measurements was $1.39 \mathrm{~ns}$. Therefore, the condition where the pulse width with dispersion $t_{p_{\text {spread }}}$ must be less or equal to $t_{p_{\max }}$ (defined as $3.46 \mathrm{~ns}$ in Section III) was satisfied. It is possible to conclude that, although the antenna has some dispersive behavior, this behavior is low enough to allow separation of the reflections.

TABLE IV. SigNALS PULSE WIDTH.

\begin{tabular}{cc}
\hline Signal & Pulse width $\left(t_{p_{\text {spread }}}\right)$ \\
\hline Transmitted & $0.69 \mathrm{~ns}$ \\
Received (simulated link) & $0.8 \mathrm{~ns}$ \\
Received (measured link) & $1.39 \mathrm{~ns}$ \\
\hline
\end{tabular}



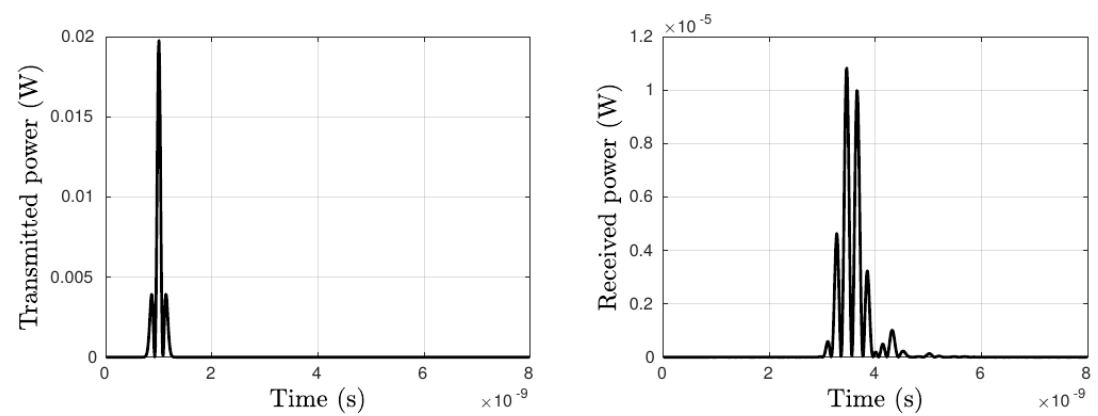

(a)
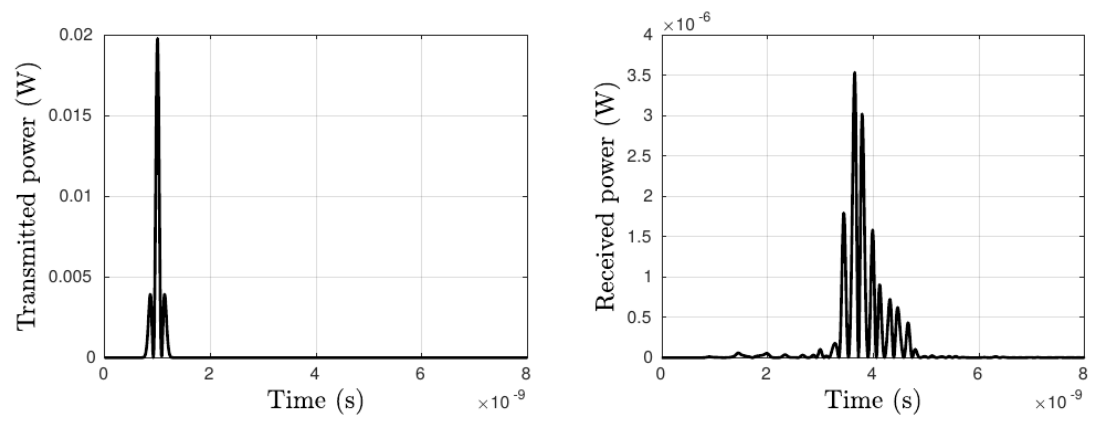

(b)

Fig. 10. Power pulses in the time-domain: (a) Simulated antenna link and (b) Measured antenna link.

\section{CONCLUSIONS}

This paper has highlighted the importance of an adequate detection of the reflected peaks measured by a GPR to improve the GPR inverse problem solution technique in multilayer problems. Consequently, we have devised a new practical step by step methodology to obtain specific antenna requirements (its operating frequency and temporal response) through the analysis of homogeneous and low-loss dielectric multilayer problems. In this way, the proposed methodology serves as a guide for GPR design to generate efficient solutions.

A test pavement problem was defined to estimate the antenna requirements using the proposed methodology. Finally, a low-cost antenna was proposed to fulfill the requirements. The validation results of the antenna showed low dispersive behavior, which indicates that there will be no overlap of the GPR reflected signals.

\section{ACKNOWLEDGMENT}

This work was supported by CAPES, Fapemig, CNPq, FUNDEP and ENACOM. We would like to thank Editage (www.editage.com) for English language editing.

\section{REFERENCES}

[1] D. Beben, W. Anigacz, and J. Ukleja, "Diagnosis of bedrock course and retaining wall using GPR," NDT \& E Int., vol. 59, pp. 77-85, 2013.

[2] J. Huisman, C. Sperl, W. Bouten, and J. Verstraten, "Soil water content measurements at different scales: accuracy of time domain reflectometry and ground-penetrating radar," J. Hydrol., vol. 245, no. 1-4, pp. 48-58, 2001.

[3] J. V. D. Kruk, S. Arcone, and L. Liu, "Fundamental and higher mode inversion of dispersed GPR waves propagating in an ice layer," IEEE Trans. Geosci. Remote Sens., vol. 45, no. 8, pp. 2483-2491, 2007.

[4] J. Q. Cai, S. X. Liu, L. Fu, and Y. Q. Feng, "Detection of railway subgrade moisture content by GPR," in 2016 16th Int. Conf. GPR, 2016. 
[5] F. M. Fernandes, A. Fernandes, and J. Pais, "Assessment of the density and moisture content of asphalt mixtures of road pavements," Constr. Build. Mater., vol. 154, pp. 1216-1225, 2017.

[6] F. A. A. Queiroz, D. A. G. Vieira, and X. L. Travassos, "Analyzing the Relevant Features of GPR Scattered Waves in Time- and Frequency-Domain," Res. Nondestruct. Eval., vol. 24, no. 2, pp. 105-123, 2013.

[7] A. Loulizi, I. L. Al-Qadi, and S. Lahouar, "Optimization of Ground-Penetrating Radar Data to Predict Layer Thicknesses in Flexible Pavements," J. Transp. Eng., vol. 129, no. 1, pp. 93-99, 2003.

[8] D. B. Oliveira, D. A. Vieira, A. C. Lisboa, and F. Goulart, "A well posed inverse problem for automatic pavement parameter estimation based on GPR data," NDT \& E Int., vol. 65, pp. 22-27, 2014.

[9] A. Loizos and C. Plati, "Accuracy of pavement thicknesses estimation using different ground penetrating radar analysis approaches," NDT \& E Int., vol. 40, no. 2, pp. 147-157, 2007.

[10] S. Lahouar and I. L. Al-Qadi, "Automatic detection of multiple pavement layers from GPR data," NDT \& E Int., vol. 41, no. 2, pp. 69-81, 2008.

[11] X. L. Travassos, S. Avila, R. Adriano, and N. Ida, "A review of ground penetrating radar antenna design and optimization,” J. Microw. Optoelectron. Electromagn. Appl., vol. 17, no. 3, pp.385-402, 2018.

[12] J. Shao, G. Fang, Y. Ji, K. Tan, and H. Yin, "A Novel Compact Tapered-Slot Antenna for GPR Applications," IEEE Antennas Wireless Propag. Lett., vol. 12, pp. 972-975, 2013.

[13] A. Ahmed, Y. Zhang, D. Burns, D. Huston, and T. Xia, "Design of UWB Antenna for Air-Coupled Impulse GroundPenetrating Radar," IEEE Geosci. Remote Sens. Lett., vol. 13, no. 1, pp. 92-96, 2016.

[14] K.-B. Ng, C. H. Chan, K.-M. Luk, "Low-cost vertical patch antenna with wide axial-ratio beamwidth for handheld satellite communications terminals," IEEE Trans. Antennas Propag., vol. 63, no. 4, pp. 1417-1424, 2015.

[15] A. S. Arezoomand, M. Naser-Moghadasi, I. Arghand, P. Jahangiri, and F. B. Zarrabi, "Photonic band gap implementation for phase centre controlling in Vivaldi antenna," IET Microw., Antennas Propag., vol. 11, no 13, pp. 1880-1886, 2017.

[16] D. B. Oliveira, A. C. Lisboa, L. A. C. Fonseca, E. J. Silva, and R. Adriano, "Low-cost antenna design for RFID readers using multiobjective optimization," Microw. Optical Technol. Lett., vol. 58, no. 4, pp. 905-908, 2016.

[17] X. L. Travassos, D. A. G. Vieira, and A. C. Lisboa, “Antenna Optimization Using Multiobjective Algorithms," ISRN Commun. Netw., vol. 2012, pp. 1-8, 2012.

[18] J. LÁČÍK, I. E. Lager, and Z. RAIDA, "Multicriteria optimization of antennas in time-domain," Radioengineering, vol. 19, no. 1, pp. 105-110, 2010.

[19] N. Telzhensky and Y. Leviatan, "Novel Method of UWB Antenna Optimization for Specified Input Signal Forms by Means of Genetic Algorithm," IEEE Trans. Antennas Propag., vol. 54, no. 8, pp. 2216-2225, 2006.

[20] H. M. Jol, Ground Penetrating Radar: Theory and Aplications. Elsevier, 2010, pp. 14-16.

[21] S. Zhao, P. Shangguan, and I. L. Al-Qadi, "Application of regularized deconvolution technique for predicting pavement thin layer thicknesses from ground penetrating radar data," NDT \& E Int., vol. 73, pp. 1-7, 2015.

[22] D. C. Nobes, "Ground penetrating radar response from voids: A demonstration using a simple model," NDT \& E International, vol. 91, pp. 47-53, 2017.

[23] IEEE Radar Definitions, IEEE Standard 686, p. 22, 2017.

[24] American National Standard of Procedures for Compliance Testing of Unlicensed Wireless Devices. ANSI Standard C63.10, p. 102, 2013.

[25] R. L. Boylestad and L. Nashelsky, Electronic devices and circuit theory. Upper Saddle River, NJ: Prentice Hall, 2002.

[26] Y. Wang, "Frequencies of the Ricker wavelet," Geophysics, vol. 80, no. 2, pp. 31-37, 2015.

[27] T. Saarenketo and T. Scullion, "Road evaluation with ground penetrating radar," J. Appl. Geophysics, vol. 43, no. 2-4, pp. 119-138, 2000.

[28] A. Benedetto and F. Benedetto, "GPR experimental evaluation of subgrade soil characteristics for rehabilitation of roads," in 9th Int. Conf. GPR, vol. 4758, pp. 708-714, 2002.

[29] S. Colagrande, D. Ranalli, and M. Tallini, "Ground Penetrating Radar Assessment of Flexible Road Pavement Degradation," Int. J. Geophysics, vol. 2011, pp. 1-11, 2011.

[30] A. Maharaj and R. Leyland, "The dielectric constant as a means of assessing the properties of road construction materials," in Proc. 29th Southern African Transport Conf. (SATC), Pretoria, pp. 487-498,2010.

[31] C. Hidalgo, C. A. Pandales, B. A. Pedroza, and M. A. Rodriguez, "Comportamiento de una pista experimental de pavimento flexible con base estabilizada con cal," Revista Ingenierias Universidad de Medellin, vol. 9, no. 16, pp. 3747, 2010.

[32] J. Y. Zhao, Z.Y. Zhang, N. W. Liu, G. Fu, and S. X. Gong,"Wideband unidirectional bowtie antenna with pattern improvement," Progress Electromagn. Res., Vol. 44, pp. 119-24, 2014.

[33] J, Yang, and A. Kishk, "The self-grounded Bow-Tie antenna," in IEEE Int. Symp. Antennas Propag. (APSURSI), pp. 1452-1455, 2011.

[34] A. A., Omar, A. Qaroot, and M. C. Scardelletti, "UWB coplanar-waveguide-fed spiral slot antenna," in 7th Eur. Conf. Antennas Propag. (EuCAP), pp. 2901-2902, 2013.

[35] M. A., Karim, M. F., Malek, M. F., Jamlos, L. Y., Seng, and N., Saudin, "Design of ground penetrating radar antenna for buried object detection," in IEEE Int. RF Microw. Conf. (RFM), pp. 253-257, 2013.

[36] K., Kim, and W. R., Scott Jr, “A resistive linear antenna for ground-penetrating radars," in Detection and Remediation Technologies for Mines and Minelike Targets IX, Vol. 5415, pp. 359-370, 2004.

[37] B. Kasi and C. K. Chakrabarty, "Ultra-Wideband Antenna Array Design For Target Detection," Progress Electromagn. Res. C, vol. 25, pp. 67-79, 2012.

[38] A. N. D. S. Jose, M. V. A. Contreras, R. Adriano, U. D. C. Resende, and J. F. Mologni, "Uncertainties minimization in open environment antenna gain estimations," in 2017 IEEE 3rd Global Electromagn. Compat. Conf. (GEMCCON), 2017. 